\title{
Reducing Delirium in Patients with COVID-19
}

Allison Brewer, MOT/S, Amy Carver, MOT/S, Allison Nance, MPH, MOT/S, Mallori Rodrigue, MOT/S, Olivia Smith, MOT/S Faculty Advisor: Anita Mitchell, PhD, OTR, FAOTA

Practitioner-Mentor: Mary Matthies, OTR/L

\begin{tabular}{|c|c|}
\hline PICO QUESTION \\
\hline $\begin{array}{c}\text { What occupation-based interventions are effective in decreasing } \\
\text { delirium in patients with COVID-19? }\end{array}$ \\
\hline BACKGROUND \& RATIONALE \\
\hline Bignificant rise in patients with COVID-19 experiencing \\
symptoms of delirium within the last year
\end{tabular}

\begin{tabular}{|c|c|c|c|c|}
\hline \multicolumn{5}{|c|}{ MAIN FINDINGS } \\
\hline $\begin{array}{l}\text { Level of } \\
\text { Evidence }\end{array}$ & Citation & Intervention & $\begin{array}{l}\text { Outcome } \\
\text { Measures }\end{array}$ & Results \\
\hline & $\begin{array}{l}\text { Khan et al. (2020) } \\
\text { Quality Score: } \\
70 \%\end{array}$ & $\begin{array}{l}120 \text { minutes a day of slow-tempo music } \\
\text { Audiobooks }\end{array}$ & CAM & \pm \\
\hline & $\begin{array}{l}\text { Martinez et al. } \\
\text { (2012) } \\
\text { Quality Score: } \\
88 \%\end{array}$ & $\begin{array}{l}\text { Multicomponent intervention delivered } \\
\text { by family members: } \\
\text { - Provision of a clock in the room } \\
\text { - Extended visitation times } \\
\text { Presence of familiar objects in the } \\
\text { r Reom }\end{array}$ & CAM & + \\
\hline Level I & $\begin{array}{l}\text { Álvarez et al. } \\
\text { (2017) } \\
\text { Quality Score: } \\
88 \%\end{array}$ & $\begin{array}{l}\text { Early and intensive OT interventions: } \\
\text { - Positioning to prevent } \\
\text { edema and pressure sores } \\
\text { - Activities of daily living } \\
\text { - Family participation } \\
\text { - Cognitive stimulation } \\
\text { Non-pharmacologic strategies: } \\
\text { - Family education } \\
\text { - Clock and calendar } \\
\text { - Avoidance of sensory deprivation } \\
\text { - Familiar objects } \\
\text { - Reorientation of patients } \\
\text { Extended visitation times }\end{array}$ & CAM & \pm \\
\hline Level II & $\begin{array}{l}\text { Mudge et al. } \\
(2013) \\
\text { Quality Score: } \\
80 \%\end{array}$ & $\begin{array}{l}\text { - Multisensory diversion and } \\
\text { relaxation strategies } \\
\text { - Reorientation } \\
\text { - De-escalate agitation } \\
\text { - Encourage independence } \\
\text { - Putrition and hydration } \\
\text { Patient/caregiver information }\end{array}$ & $\begin{array}{l}\text { AMT } \\
\text { CAM } \\
\text { MMSE }\end{array}$ & + \\
\hline Level III & $\begin{array}{l}\text { Chong et al. } \\
(2013) \\
\text { Quality Score: } \\
64 \%\end{array}$ & $\begin{array}{l}\text { Bright-light therapy (2000-3000 lux) for } \\
\text { four hours daily as part of a } \\
\text { multicomponent intervention }\end{array}$ & $\begin{array}{c}\text { DRS } \\
\text { CMMSE } \\
\text { MBI }\end{array}$ & + \\
\hline
\end{tabular}

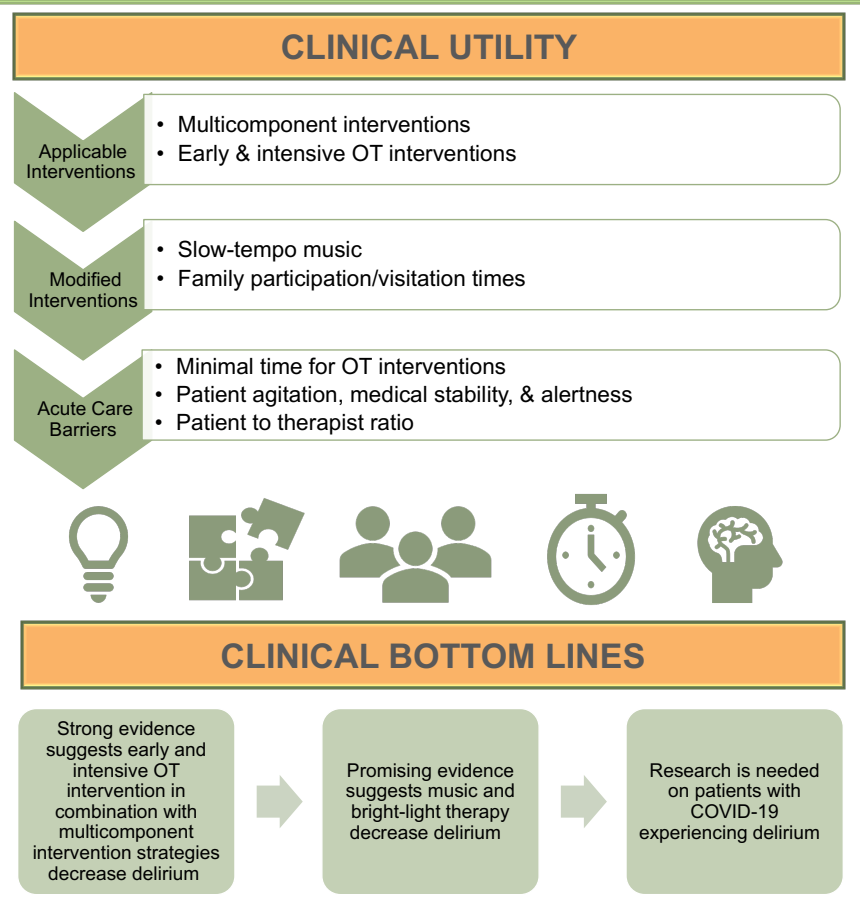

Note: OT = occupational therapy; $+=$ significant improvement; $\pm=$ non-significant improvement; $\quad$ CAM $=$ Confusion Assessment Method; DRS = Delirium Rating Scale; AMT = Abbreviated Mental Test; MMSE
$=$ Mini Mental State Exam; CMME = Chinese Mini Mental State Exam; MBI = Modified Barthel Index

\begin{tabular}{|c|c|c|c|c|}
\hline \multicolumn{4}{|c|}{ LIMITATIONS } \\
\hline $\begin{array}{c}\text { Small sample } \\
\text { size }\end{array}$ & $\begin{array}{c}\text { Data not adjusted } \\
\text { to physiological } \\
\text { stress }\end{array}$ & $\begin{array}{c}\text { Simple data } \\
\text { masking }\end{array}$ & $\begin{array}{c}\text { Small number of } \\
\text { patients per room }\end{array}$ & $\begin{array}{c}\text { Conflict of } \\
\text { interest }\end{array}$ \\
\hline Attrition & $\begin{array}{c}\text { Not cost- } \\
\text { effective }\end{array}$ & No control group & $\begin{array}{c}\text { Groups not } \\
\text { randomly } \\
\text { selected }\end{array}$ & $\begin{array}{c}\text { Risk of observer } \\
\text { bias }\end{array}$ \\
\hline
\end{tabular}

\begin{tabular}{|c|c|}
\hline \multicolumn{2}{|c|}{ RECOMMENDATIONS } \\
\hline \multicolumn{2}{|c|}{$\begin{array}{l}\text { All interventions should be implemented with } \\
\text { caution monitoring the effectiveness for patients } \\
\text { with COVID-19. }\end{array}$} \\
\hline \multicolumn{2}{|c|}{$\begin{array}{l}\text { Implementation of the interventions as } \\
\text { frequently as possible. }\end{array}$} \\
\hline TRACKER TOOL & REFERENCES \\
\hline 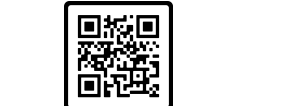 & 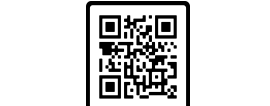 \\
\hline (8) SCAN ME & (8)SCAN ME \\
\hline
\end{tabular}

